\title{
Mass Spectrometry Parameters Optimization for the 46 Multiclass Pesticides Determination in Strawberries with Gas Chromatography Ion-Trap Tandem Mass Spectrometry
}

\author{
Virgínia C. Fernandes, ${ }^{1,2}$ Jose L. Vera, ${ }^{1,3}$ Valentina F. Domingues, ${ }^{1}$ Luís M. S. Silva, ${ }^{4}$ \\ Nuno Mateus, ${ }^{2}$ Cristina Delerue-Matos ${ }^{1}$ \\ ${ }^{1}$ Requimte, Instituto Superior de Engenharia, Instituto Politécnico do Porto, Rua Dr António Bernardino de Almeida, 431, \\ 4200-072 Porto, Portugal \\ ${ }^{2}$ CIQ- Centro de Investigação em Química, Departamento de Química e Bioquímica, Faculdade de Ciências, Universidade do \\ Porto, Porto, Portugal \\ ${ }^{3}$ Requimte, Faculdade de Ciências, Universidade do Porto, Porto, Portugal \\ ${ }^{4}$ CIETI, Instituto Superior de Engenharia, Instituto Politécnico do Porto (ISEP), Porto, Portugal
}

\begin{abstract}
Multiclass analysis method was optimized in order to analyze pesticides traces by gas chromatography with ion-trap and tandem mass spectrometry (GC-MS/MS). The influence of some analytical parameters on pesticide signal response was explored. Five ion trap mass spectrometry (IT-MS) operating parameters, including isolation time (IT), excitation voltage (EV), excitation time $(\mathrm{ET})$, maximum excitation energy or "q" value (q), and isolation mass window (IMW) were numerically tested in order to maximize the instrument analytical signal response. For this, multiple linear regression was used in data analysis to evaluate the influence of the five parameters on the analytical response in the ion trap mass spectrometer and to predict its response. The assessment of the five parameters based on the regression equations substantially increased the sensitivity of IT-MS/MS in the MS/MS mode. The results obtained show that for most of the pesticides, these parameters have a strong influence on both signal response and detection limit. Using the optimized method, a multiclass pesticide analysis was performed for 46 pesticides in a strawberry matrix. Levels higher than the limit established for strawberries by the European Union were found in some samples.
\end{abstract}

Key words: Optimization parameters, Multiclass pesticides, GC-MS/MS, Ion trap, Strawberries

\section{Introduction}

$\mathrm{P}$ esticides are used to protect crops before and after harvest from infestation by pests and plant diseases. A

Electronic supplementary material The online version of this article (doi:10.1007/s13361-012-0488-6) contains supplementary material, which is available to authorized users.

Correspondence to: Valentina F. Domingues; e-mail: vfd@isep.ipp.pt consequence of their use may be the presence of pesticide residues in treated products, fruits, vegetables, grains, and other commodities. Even after being washed, stored, processed, and prepared, some pesticide residues may remain in both fresh products and processed foods. Therefore, multiresidual methodologies that can determine a large number of pesticides simultaneously with satisfactory sensitivity and selectivity are highly required [1]. Stringent international food safety standards require monitoring some chemicals in a variety of food samples 
at trace level with the lowest maximum residue limit (MRL) being set at $10 \mathrm{ng} / \mathrm{g}$ (UE) [2, 3]. Gas chromatography coupled to an electron capture detector (GCECD), a nitrogen phosphorus detector, and different other detectors are still widely used as analytical technique for the trace analysis of pesticides in various environmental and food matrices with high sensitivity [4-13]. Nowadays, the use of mass spectrometry (MS) detection for a proper and accurate analyte identification together with the electron ionization (EI) mode and the GC retention time information is essential [2, 14-16]. Ion trap (IT) GC-MS has the potential to identify analytes at trace levels and to avoid the influence of matrix components as well as allows for selective analysis by MS/MS. This could be achieved by collision-induced dissociation (CID) of a selected unique precursor ion that produces ions in sufficient abundance ratios specific to the detection of a particular molecule [2]. In order to attain high sensitivity to achieve low detection limits, the instrumental parameters of IT-MS affecting the performance of this system must be thoroughly optimized [14]. Although parameters for some compounds are available in MS/MS libraries, the optimization of GC-MS/MS parameters is indispensable in order to run with best efficiency [17-20]. There are a few papers describing the optimization and the best parameters for each analyte in a given apparatus [2]. The optimization of GC-MS/MS system requires assessing IT-MS parameters influence by the approach of changing one-factor-at-a-time (OFAT) [2]. This approach does not give information on interactions between factors, so it can miss the optimal settings when interactions do occur. Statistically designed experiments such as Plackett-Burman and central composite designs can help to optimize analytical parameters much more efficiently and in less experimental runs [19]. Optimization of MS/MS parameters is a hard work for each analyte; hence, knowledge of optimal values will save considerable time in analytical method development.

The aim of the present study was to establish an overall analytical method and optimization of a set of instrumental parameters in order to attain the highest possible sensitivity for pesticides determination. Particular attention was paid to the optimization of five IT-MS parameters, namely, the duration of the ion isolation waveform voltage [isolation time (IT)], the duration of the ion excitation [excitation time (ET)], the mass range window around the ion of interest [isolation mass window (IMW)], excitation voltage (EV) and the maximum excitation energy (q), which is defined as the amount of energy that holds a precursor ion in the ion trap during excitation.

In this work a visual basic for macro applications in Microsoft Excel was created to compute the signal response based on the best regression model for all possible combinations among the five parameters in a total of 2160 arrangements and save the maximum value. Bearing all this in mind, a GC-MS/MS based multiresidue analysis method was developed covering 46 pesticides, including pyrethroids, organophosphorus, carbamates, strobilurines, pyrimidines, thiophthalimides, dinitrophenols derivative, azoles, anilides, dicarboximides, anilinopyrimidines, aryloxyphenoxy propionic acids, 2,6-dinitroanilines, triazines and a set of persistent ones such as organochlorines. The optimized method was then used in the screening program and the levels of these pesticides in strawberries are reported in this paper.

\section{Materials and Methods}

\section{Chemicals and Preparation of Standard Solutions}

Reference standards were purchased from Sigma Aldrich (Steinheim, Germany), Riedel-de Häen (Seelze, Germany), and Chem Service (West Chester, PA, USA). Stock standard solutions (approximately $2000 \mu \mathrm{g} . \mathrm{L}^{-1}$ ) were prepared by dissolving reference standards in $\mathrm{n}$ hexane and methanol and were stored in a freezer at $4^{\circ}$ C. Working pesticide standard mixtures were prepared by dilution of stock solutions in n-hexane. n-Hexane and methanol were chromatographic grade and were supplied by Merck (Darmstadt, Germany).

\section{Apparatus}

GC-MS/MS instrument, TRACE GC Ultra (Thermo Fisher Scientific, Austin, TX, USA) gas chromatograph coupled with a Polaris $\mathrm{Q}$ ion trap mass spectrometer was used. The system included an AS-3000 autosampler. A ZBXLB capillary column from Phenomenex (30 $\mathrm{m} \times$ $0.25 \mathrm{~mm} \times 0.25 \mu \mathrm{m}$ ) was used for chromatographic separation. The system was controlled by Xcalibur software, ver. 1.3. The interface line and ion source temperatures were maintained at $250{ }^{\circ} \mathrm{C}$ in all experiments. Injections $(2 \mu \mathrm{L})$ were done in the splitless mode. MS/MS conditions such as isolation (wideband application (IMW), isolation time (IT)), fragmentation (excitation time (ET) and voltage (EV) and factor "q" were optimized for each analyte, beginning with the following base conditions: factor $\mathrm{q}=0.45, \mathrm{EV}=1 \mathrm{~V}$, IT $=12 \mathrm{~ms}$, $\mathrm{ET}=15 \mathrm{~ms}, \mathrm{IMW}=1$ and carrier flow $1.3 \mathrm{~mL} \mathrm{~min}^{-1}$.

\section{Gas Chromatograph Conditions}

The column oven temperature was programmed as follows: initial temperature $40^{\circ} \mathrm{C}$ (held for $1 \mathrm{~min}$ ), increased by $30^{\circ}$ $\mathrm{C} / \mathrm{min}$ to $220^{\circ} \mathrm{C}$ (held for $5 \mathrm{~min}$ ), increased by $10^{\circ} \mathrm{C} / \mathrm{min}$ to $250{ }^{\circ} \mathrm{C}$ and held at this temperature for $20 \mathrm{~min}$ and finally increased again by $5{ }^{\circ} \mathrm{C} / \mathrm{min}$ to $285{ }^{\circ} \mathrm{C}$ and held at this temperature for $5 \mathrm{~min}$. The mass spectrometer was operated in electron ionization (EI) mode at $70 \mathrm{eV}$ with an external ionization source. The inlet temperature was $240{ }^{\circ} \mathrm{C}$ and helium (purity $\geq 99.999 \%$ ) was used as carrier gas at $1 \mathrm{~mL} /$ min and the injection volume was $2 \mu \mathrm{L}$. The ion source 
temperature was $250{ }^{\circ} \mathrm{C}$ and the electron multiplier was operated at $2100 \mathrm{~V}$ (autotune to gain of $1 \times 10^{7}$ ).

\section{Limit of Detection}

The limit of detection (LOD), also defined as the lowest concentration that the analytical process can differentiate from background levels, was estimated for a signal-to-noise ratio $(\mathrm{S} / \mathrm{N})$ of three from the chromatograms analysis.

\section{Multiple Linear Regression Analysis}

For each pesticide, multiple linear regression tests were conducted in order to estimate the relationship between the statistically significant parameters of IT-MS/MS detection and the instrumental signal response by fitting a linear equation to observed data.

\section{Macro Edition in Microsoft Excel}

A visual basic for macro applications (VBA) was developed in Microsoft Excel in order to assess the maximum of $8 \mathrm{ET} \times$ $6 \mathrm{IT} \times 5 \mathrm{EV} \times 3 \mathrm{q} \times 3$ IMW combinations, available in the ITMS/MS detector, based on the predicted value determined by the multiple regression equation.

\section{Sampling}

Strawberries were collected in the first week of May in 2009 and 2010 from crops in the center of Portugal. Different varieties of strawberries were collected including Siba, Camarosa, Festival, and Albion. Samples determined to be without any target analytes were used as blank strawberry samples. The samples were analyzed following the procedure described below.

Extraction and Cleanup Procedures The selected QuEChERS (EUMIV50CT-VP) and cleanup (CUMPS15C18CT) were obtained from UCT (Bristol, PA, USA). For the initial extraction step, an amount (10 g) of chopped strawberries was weighted into a $50 \mathrm{~mL}$ centrifuge tube and $50 \mu \mathrm{L}$ of internal standard (IS) solution was added. The strawberry sample was left during $30 \mathrm{~min}$ at room temperature to let the $n$-hexane evaporate before the addition of $10 \mathrm{~mL}$ of acetonitrile.

The resulting solution was shaken for $1 \mathrm{~min}$ followed by the addition of $6 \mathrm{~g}$ anhydrous magnesium sulfate, $1.5 \mathrm{~g}$ sodium chloride, $1.5 \mathrm{~g}$ trisodium citrate dihydrate, and $0.75 \mathrm{~g}$ disodium hydrogenocitrate sesquihydrate. The centrifuge tube was capped and shaken vigorously for 1 min to prevent salt agglomeration before centrifugation at $3000 \mathrm{rpm}$ for $5 \mathrm{~min}$ at room temperature. An aliquot of $1.5 \mathrm{~mL}$ was sampled from the upper layer into a $2 \mathrm{~mL}$ cleanup vial containing $150 \mathrm{mg}$ primary secondary amine (PSA), $150 \mathrm{mg} \mathrm{MgSO}_{4}$, and $50 \mathrm{mg} \mathrm{C} 18$ and again vortexed for $1 \mathrm{~min}$ and then centrifuged for $5 \mathrm{~min}$ at $4000 \mathrm{rpm}$ at room temperature. From the upper layer of the prepared sample, an aliquot of $1.0 \mathrm{~mL}$ was transferred into a vial and put under a mild stream of nitrogen to dryness. Finally, $1 \mathrm{~mL}$ of $\mathrm{n}$-hexane was added to dissolve the residue and then $2 \mu \mathrm{L}$ of this solution was injected onto the gas chromatograph.

For fortification studies, $10 \mathrm{~g}$ of strawberry sample free of detectable pesticides were spiked and homogenized at 50, 200, and $400 \mu \mathrm{g} \cdot \mathrm{kg}^{-1}$ levels for each pesticide. In the case of organochlorine pesticides 30 , 90 , and $180 \mu \mathrm{g} \cdot \mathrm{kg}^{-1}$ levels were used. The mixture was left for $30 \mathrm{~min}$ before the beginning of the extraction process. Samples were then prepared according to the procedure aforementioned.

\section{Results and Discussion}

The optimization of MS/MS parameters in IT-MS was carried out in four steps: (1) isolation of precursor ion and subsequent product ion selection, (2) screening analysis, (3) multiple linear regression test to check the importance of each parameter on the signal response, fitting data to linear and second order models for the significant parameters and model discrimination, and finally (4) combinatorial optimization based on the best previously chosen model.

\section{Isolation of Precursor Ion and Product Ions Selection}

The most abundant ion from the spectra of the different pesticides was selected as precursor ion and it was then isolated in the ion trap and fragmented by collision induced dissociation (CID). The two most abundant product ions, $\mathrm{Q}_{1}$ and $\mathrm{Q}_{2}$ [1] were selected and monitored and the spectrum was obtained with the default operating parameters from the GC-MS/MS system (IT=12 ms, ET=15 ms, IMW=1, q= $0.45, \mathrm{EV}=1.0 \mathrm{~V})($ Table 1).

\section{Screening Analysis}

Different values of EV, ET, IMW, q, and IT were set up by a one-factor-at-a-time (OFAT) method, meaning that when one parameter is changed the others are fixed at their default value. Regarding a multiclass pesticides signal maximization, the search of the best IT-MS parameters is necessary. Thus, the parameters were varied in order to determine the suitable values at which both absolute peak area of the product ions and signal to noise $(\mathrm{S} / \mathrm{N})$ ratio were maximized for the 46 pesticides under investigation. The concentration of the standard mixture solution used in this work was $150 \mu \mathrm{g} . \mathrm{L}^{-1}$. The results obtained for the signal to noise ratio by OFAT experiments are shown in Figure 1. An example of OFAT study (HCB) concerning the dependence of $\mathrm{q}$ factor on area response is shown in Figure S1 (Supplementary Information). An important 
Table 1. Precursor Ions and the Products Ions $\left(\mathrm{Q}_{1}\right.$ and $\left.\mathrm{Q}_{2}\right)$ for the Selected Pesticides

\begin{tabular}{|c|c|c|c|c|c|c|}
\hline Chemistry class & Pesticides & CAS & MW $(\mathrm{g} / \mathrm{mol})$ & Ion precursor & $\mathrm{Q}_{1}$ & $\mathrm{Q}_{2}$ \\
\hline Chloroacetanilide & Alachlor & $15972-60-8$ & 270 & 188 & 160 & 132 \\
\hline \multirow{12}{*}{ Organochlorine } & Aldrin & $309-00-2$ & 365 & 263 & 227 & 193 \\
\hline & Dieldrin & $60-57-1$ & 381 & 243 & 211 & 176 \\
\hline & Endrin & $72-20-8$ & 381 & 245 & 209 & 173 \\
\hline & $\mathrm{HCB}$ & $118-74-1$ & 285 & 284 & 214 & 249 \\
\hline & Lindane & $58-89-9$ & 291 & 183 & 109 & 181 \\
\hline & $o, p^{\prime}-\mathrm{DDT}$ & $789-02-6$ & 355 & 235 & 165 & 199 \\
\hline & $\mathrm{p}, \mathrm{p}^{\prime}-\mathrm{DDD}$ & $72-54-8$ & 320 & 235 & 165 & 199 \\
\hline & $\mathrm{p}, \mathrm{p}^{\prime}-\mathrm{DDE}$ & $72-55-9$ & 318 & 318 & 299 & 281 \\
\hline & Methoxychlor & $72-43-5$ & 346 & 227 & 169 & 197 \\
\hline & $\alpha, \beta, \delta-\mathrm{HCH}$ & $608-73-1$ & 291 & 183 & 179 & 177 \\
\hline & $\alpha$-Endosulfan & $959-98-8$ & 407 & 195 & 191 & 170 \\
\hline & $\beta$-Endosulfan & $33213-65-9$ & 407 & 195 & 191 & 170 \\
\hline \multirow{3}{*}{ Triazines } & Atrazine desethyl & $6190-65-4$ & 188 & 172 & 136 & 145 \\
\hline & Atrazine & $1912-24-9$ & 216 & 200 & 164 & 122 \\
\hline & Simazine & $122-34-9$ & 202 & 200 & 172 & 164 \\
\hline Strobin & Azoxystrobin & $131860-33-8$ & 403 & 344 & 327 & 325 \\
\hline \multirow[t]{8}{*}{ Pyrethroids } & $\beta$-Cyfluthrin & $68359-37-5$ & 434 & 163 & 91 & 127 \\
\hline & Bifentrin & $82657-04-3$ & 423 & 181 & 165 & 164 \\
\hline & Cypermethrin & $52315-07-8$ & 416 & 181 & 163 & 152 \\
\hline & Deltametrin & $52918-63-5$ & 505 & 172 & 141 & 88 \\
\hline & $\lambda$-Cyhalothrin & $68085-85-8$ & 450 & 181 & 152 & 141 \\
\hline & Permethrin & $52645-53-1$ & 391 & 183 & 165 & 153 \\
\hline & Fenvalerate & $51630-58-1$ & 420 & 125 & 121 & 132 \\
\hline & Fenpropathrin & $39515-41-8$ & 349 & 181 & 152 & 153 \\
\hline \multirow{2}{*}{ Thiophthalimide } & Captan & $133-06-2$ & 301 & 264 & 257 & 254 \\
\hline & Folpet & $133-07-3$ & 297 & 260 & 151 & 189 \\
\hline \multirow[t]{3}{*}{ Organophosphorus } & Chlorpyrifos & $2921-88-2$ & 351 & 197 & 114 & 148 \\
\hline & Malathion & $121-75-5$ & 330 & 173 & 134 & 106 \\
\hline & Diazinon & $333-41-5$ & 304 & 179 & 167 & 110 \\
\hline \multirow[t]{4}{*}{ Pyrimidine } & Cyprodinil & $121552-61-2$ & 225 & 224 & 208 & 197 \\
\hline & Bupirimate & $121552-61-2$ & 316 & 208 & 178 & 129 \\
\hline & Mepanipyrim & $110235-47-7$ & 223 & 222 & 207 & 221 \\
\hline & Pyrimethanil & 53112-28-0 & 199 & 198 & 183 & 158 \\
\hline Unclassified & Dazomet & $533-74-4$ & 162 & 162 & 89 & 44 \\
\hline Anilide & Fenhexamid & $126833-17-8$ & 302 & 177 & 166 & 153 \\
\hline Unclassified & Fludioxinil & 131341-86-1 & 248 & 248 & 235 & 191 \\
\hline$N$-methyl Carbamate & Methiocarb & $2032-65-7$ & 225 & 168 & 153 & 109 \\
\hline 2,6-Dinitroaniline & Pendimethalin & 40487-42-1 & 281 & 252 & 191 & 162 \\
\hline \multirow[t]{2}{*}{ Aryloxyphenoxy propionic acid } & Quizalofop-p-ethyl & $100646-51-3$ & 299 & 223 & 151 & 255 \\
\hline & Fluazifop-p-butyl & $79241-46-6$ & 383 & 254 & 249 & 163 \\
\hline \multirow[t]{2}{*}{ Azole } & Tetraconazole & $112281-77-3$ & 372 & 336 & 249 & 105 \\
\hline & Myclobutanil & 88671-89-0 & 289 & 152 & 150 & 151 \\
\hline Phenylsulfamide & Tolylfluanid & $731-27-1$ & 347 & 138 & 137 & 136 \\
\hline \multirow[t]{3}{*}{ Dicarboximide } & Vinclozolin & 50471-44-8 & 286 & 212 & 172 & 145 \\
\hline & Procymidone & $32809-16-8$ & 284 & 283 & 281 & 282 \\
\hline & Iprodione & 36734-19-7 & 330 & 314 & 271 & 153 \\
\hline
\end{tabular}

increase of area was observed when " $q$ " factor increases from 0.225 to 0.45 (Figure 1q). This increase is more remarkable for $\mathrm{HCB}$, vinclozolin and bifenthrin. In particular, bifenthrin achieved a LOD of $1.61 \mu \mathrm{g} . \mathrm{L}^{-1}$ for $\mathrm{q}=$ 0.255 and a LOD of $0.49 \mu \mathrm{g} . \mathrm{L}^{-1}$ for $\mathrm{q}=0.45$, whereas HCB attained higher sensitivity as " $q$ " rose with a LOD decrease from $11.25 \mu \mathrm{g} \cdot \mathrm{L}^{-1}$ to $0.68 \mu \mathrm{g} \cdot \mathrm{L}^{-1}$.

Figure 1 (IMW) shows a variation in signal response of $\mathrm{HCB}$, diazinon, pyrimethanil, and tolylfluanid as a result of IMW change.

In the case of IT, the variation in signal response was more pronounced for diazinon, bifenthrin, alachlor, and tolylfluanid [Figure 1(IT)]. The S/N values for pyrimethanil and cyprodinil showed a maximum for IT $=12 \mathrm{~ms}$.
As to $\mathrm{EV}$ dependence, the pesticides with higher variation in signal to noise value were diazinon, bifenthrin, alachlor, pendimethaline, and tolylfluanid [Figure 1(EV)].

As shown in Figure 1(ET), some pesticides (diazinon, bifenthrin, DDT, pyrimidine pesticides, pendimethaline, tolylfluanid, and myclobutanil) revealed a random variation with respect to ET influence.

Hence, for some pesticides, it seems that better LODs can be achieved by changing the IMW to detect neighboring ions or by varying other parameters of the ion trap (i.e., IT, ET, EV, or q).

This screening analysis proves that the variation of one parameter can mean a change in the response, so the importance of applying statistical studies may allow the prediction of the best parameter conditions. 

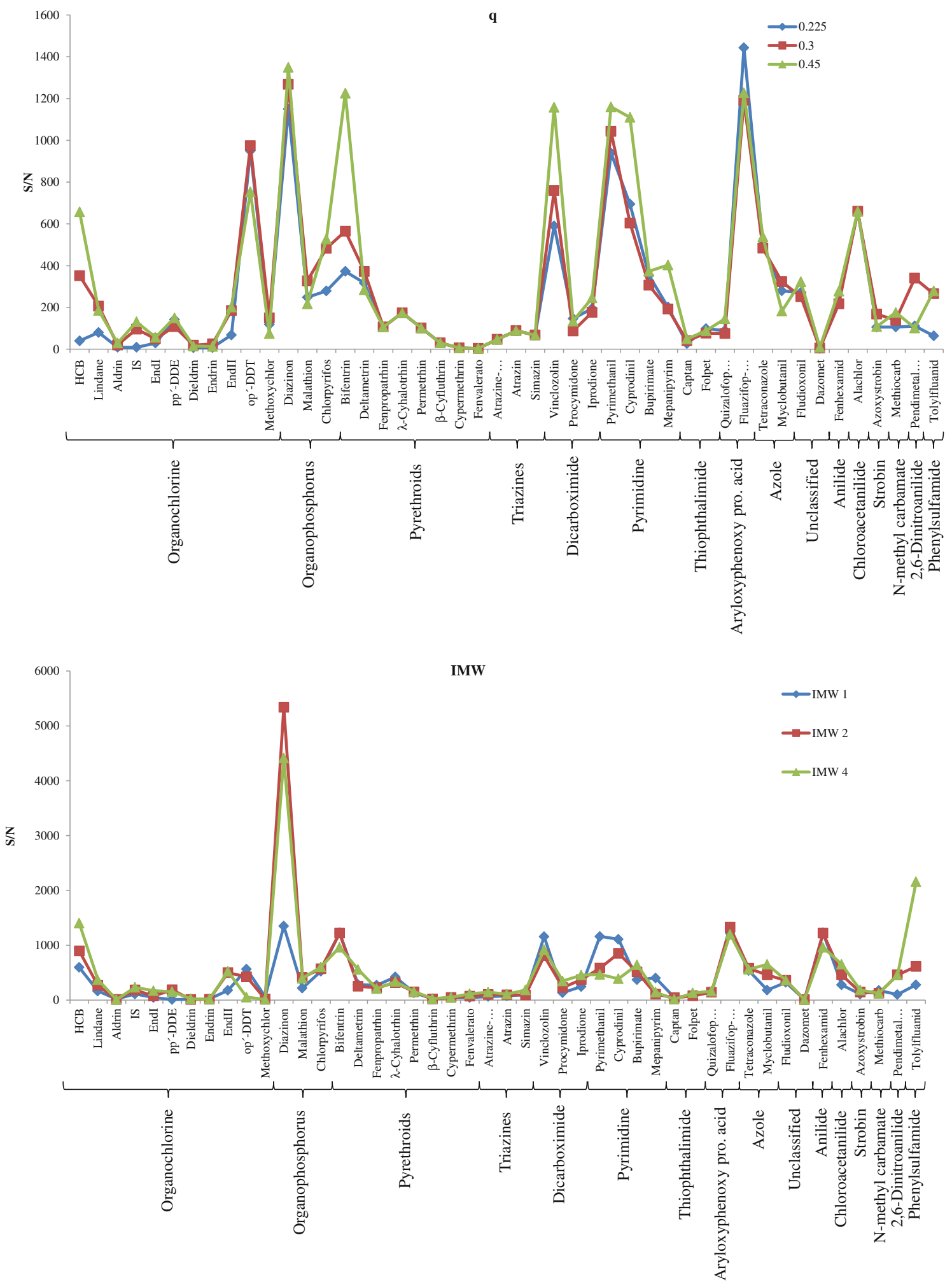

Figure 1. Plot of average signal to noise $(\mathrm{S} / \mathrm{N})$ versus, " $q$ " value, IMW, IT, ET, and EV for the 46 multiclass pesticides

\section{Multiple Linear Regression}

Multiple linear regression tests were carried out in order to estimate the influence of each parameter up to five on the IT-MS analytical response. In most cases, the true functional relationship is unknown. This study allowed finding the parameters that have significant 

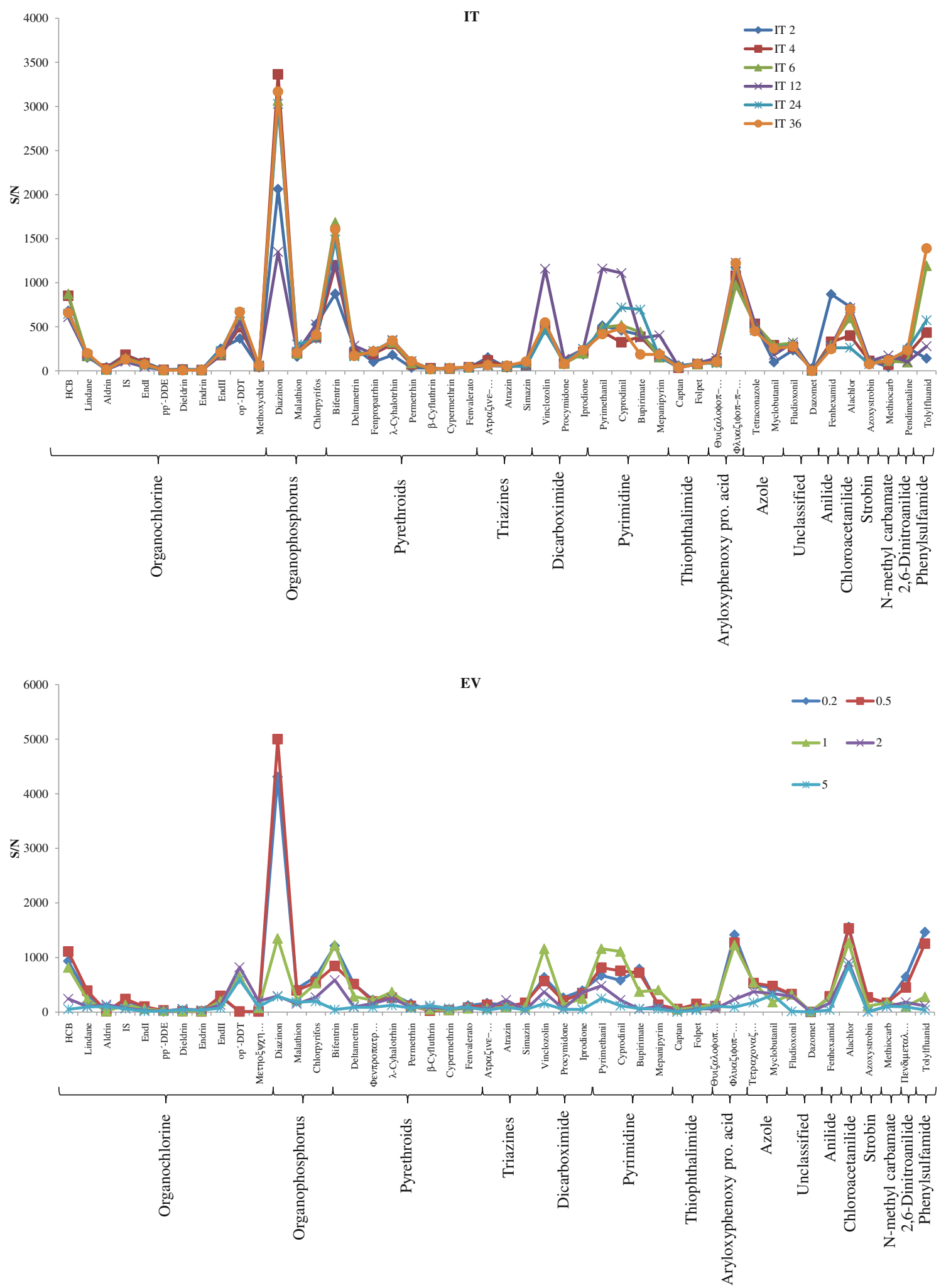

Figure 1. (continued)

influence on signal response. Hence the IMW, IT, ET, $\mathrm{EV}$, and $q$ values were screened in order to discard the irrelevant parameters keeping the remaining for subsequent optimization.
Model Discrimination To evaluate the "best" empirical regression equation, the analytical responses obtained were fitted to linear models that included only first order terms (Equation 1), and both first and second-order (curvature) terms (Equation 2). 


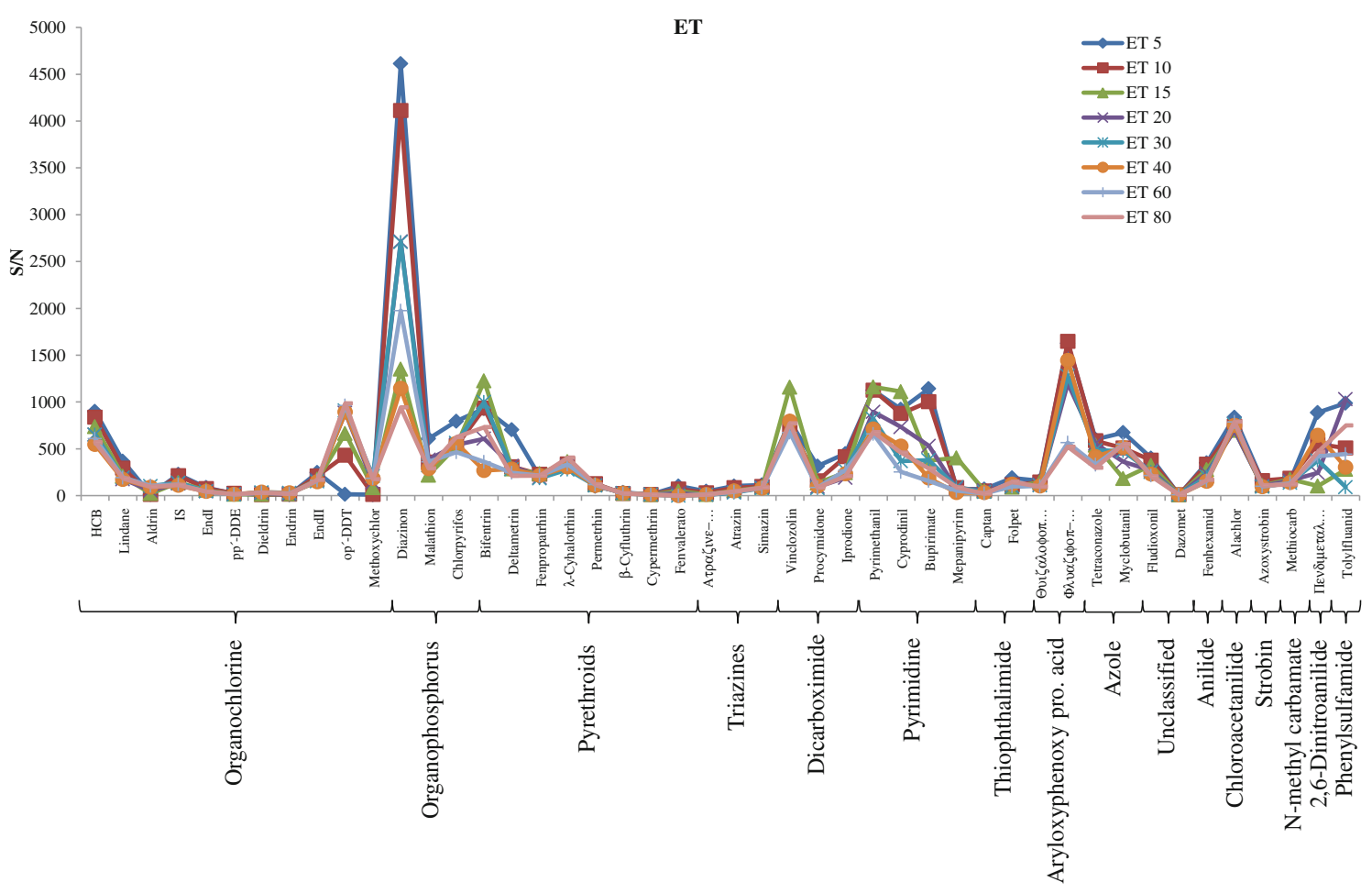

Figure 1. (continued)

The regression equations for the analytical response $(\mathrm{Y})$ in terms of the factors $\left(\mathrm{x}_{\mathrm{i}}\right)$ are as follows:

$$
\begin{gathered}
\text { First order : } Y=\beta_{0}+\sum_{i=1}^{n} \beta_{i} x_{i} \\
\text { Second order : } Y=\beta_{0}+\sum_{i=1}^{n}\left(\beta_{i} x_{i}+\beta_{i i} x_{i}^{2}\right)
\end{gathered}
$$

where $\beta_{0}$ is the independent coefficient; $n$ refers to the number of significant factors to a maximum of $5, x_{i}$ are the values of IT-MS parameters (factors), $\beta_{i}$ represent the coefficients for the main effect; and $\beta_{i j}$ are the coefficients for the second order effect where $i=1,2,3,4$ and 5 stands for IMW, IT ET, EV, and q, respectively.

Test for Significance of Regression The test for significance of regression is a statistical test that checks if there is a linear relationship between the response variable $y$ and a subset of the regressor variables $x_{1}, x_{2}, \ldots, x_{k}$. The test procedure involves an analysis of variance partitioning the total sum of squares $\mathrm{SS}_{\mathrm{T}}$ into a sum of squares due to the model (or to regression) and a sum of squares due to the residual (or error), say:

$$
S S_{T}=S S_{R}+S S_{E}
$$

Based on the theory for discrimination of nested models described in textbooks $[21,22]$, a comparison between first and second order models was performed. A kind of a likelihood test ratio adapted for regression models was conducted. For this, a statistic $T$ was computed as follows:

$$
T=\frac{S S_{E 1}-S S_{E 2}}{S S_{E 2}} \cdot \frac{n-k_{2}}{k_{1}}
$$

for $n$ data points, where $k$ is the number of model coefficients and subscripts 1 and 2 refer to first and second order model, respectively. This ratio obeys to an $F$ distribution, asymptotically for large $n$, with $k_{1}$ degrees of freedom in numerator and $n-k_{2}$ degrees of freedom in denominator. For $\mathrm{T}$ values greater than $\mathrm{F}_{\text {critical }}$ the null hypothesis that states that there are no differences between the two models must be rejected. It was proven that the second order model fits the experimental data better in most of the cases with a level of significance of 0.05 as presented in Table 2. This fact implies the existence of stationary points for the model equation because of the curvature of the surface response. At the same time, the coefficients of determination $\left(\mathrm{R}^{2}\right)$ were computed for the two models and are also presented in Table 2. Although the second order model always achieved higher $\mathrm{R}^{2}$ values, the regression test proves that the ones marked by an asterisk in Table 2 are not significantly better.

Tests on Individual Regression Coefficients The chosen model might be more accurate with the deletion of one or 
Table 2. Coefficient of Determination for First and Second Order and Statistic $T$ Used for Model Discrimination

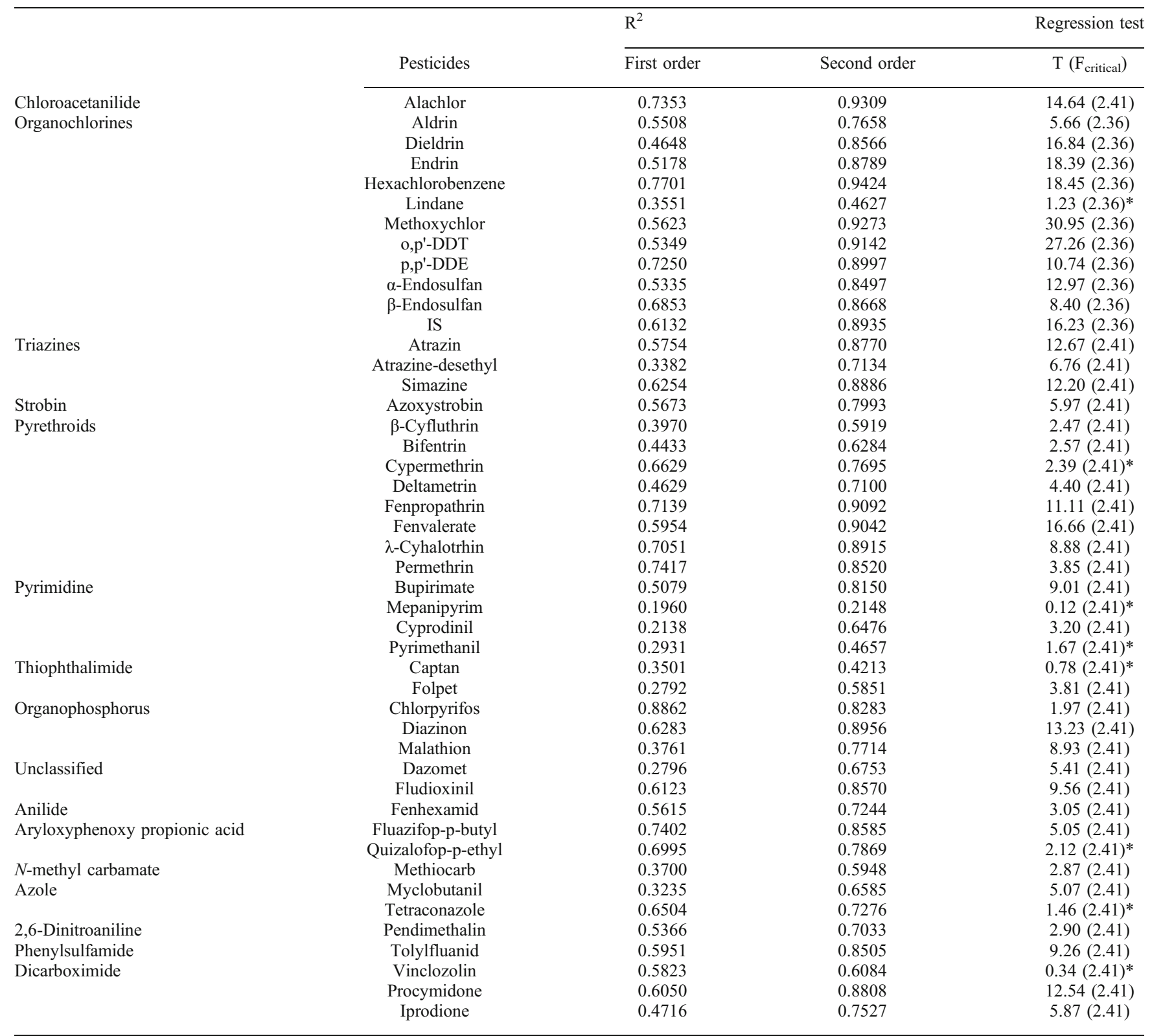

*Second order model is not significantly better than first order model

more of the coefficients belonging to the general Equations 1 or 2. The statistics for testing the significance of any individual regression coefficient is:

$$
t_{0}=\frac{\widehat{\beta}_{j}}{\widehat{\sigma}^{2} C_{j j}}
$$

where $C_{j j}$ is the diagonal element of the matrix variancecovariance $\left(\mathrm{X}^{\mathrm{T}} \mathrm{X}\right)^{-1}$ for the corresponding $\widehat{\beta}_{j}$. The denominator of the Equation 5 is often called the standard error of the regression coefficient $\widehat{\beta}_{j}$, that is:

$$
s e\left(\widehat{\beta}_{j}\right)=\sqrt{\widehat{\sigma}^{2} C_{j j}}
$$

where $\widehat{\sigma}$ refers to the standard error of regression calculated as:

$$
\widehat{\sigma}=\sqrt{\frac{S S_{E}}{n-k}}
$$

The IMW is the mass range window around the ion of interest that is isolated for optimization of MS/MS parameters. The multiple regression test proved that the IMW parameter has significant effect in the area response of 1 chloroacetanilide, 4 organochlorines, 4 pyrethrois, 1 thiophthalimide, 1 unclassified, and 1 aryloxyphenoxy propionic acid pesticides. 
The IMW parameter is not significant for triazines, strobin, pyrimidine, $n$-methyl carbamate, azole, 2,6-dinitroanilide, phenylsulfamide, and dicarboximide.

Another pertinent parameter for MS/MS optimization is the maximum excitation energy (q), which is defined as the amount of energy that holds a precursor ion in the ion trap during excitation and thus influences the usable mass range. Only three values (instrument characteristics) can be set for "q." Figure S1 shows the chromatogram of $\mathrm{HCB}$ for all acceptable q values. The area response related to $\mathrm{q}=0.45$ is 11.5 times higher than with $\mathrm{q}=0.225$. Since higher " $q$ " values allow more energy to be deposited in the precursor ion before its dissociation (CID), it can be concluded that these pesticides need more energy for fragmentation. Regarding multiple linear regression test, " $q$ " has a significant effect on the signal response for most of the organochlorine pesticides, 4 pyrethroids, 1 organophosphorus and 1 unclassified pesticide.

The isolation time (IT) of an ion is defined as the duration of the ion isolation waveform voltage applied to isolate a selected precursor ion. An initial value of $12 \mathrm{~ms}$ was imposed, which was afterward changed for optimization purposes of the $\mathrm{S} / \mathrm{N}$ ratio of individual compounds. The test on individual regression coefficients showed that more than half of the studied pesticides were affected by the variation of this parameter.

The EV parameter has significant effect in the area response of 1 chloroacetanilide, 10 organochlorines, 3 triazines, 6 pyrethroids, 1 pyrimidine, 1 thiophthalimide, 3 organophosphorus, 1 unclassified, $1 N$-methyl carbamate, 1 azole, 1 dicarboximide, 1 2,6-dinitroaniline, 1 aryloxyphenoxy propionic acid, and 1 phenylsulfamide pesticides.

As can be proven in the multiple linear regression test, the excitation time (ET) parameter has effect on the MS/MS determination of about $2 / 3$ of the pesticides tested.

The results showed that an optimum ET parameter with a value of $60 \mathrm{~ms}$ holds only for some OCPs. In these cases, the ET value of $60 \mathrm{~ms}$ is always combined with an EV value of $2 \mathrm{v}$, and both these values are the higher ones of the ET and EV range chosen. The EV and ET were significant parameters for $70 \%$ and $67 \%$ of the 46 studied pesticides, respectively.

In summary, the statistical analysis allowed a quick evaluation of the variation in the signal response of certain pesticides as a result of ion trap parameters change.

The obtained optimum conditions for the instrumental parameters were used to check the sensitivity of IT-MS. Due to the absence of background signals, high signal-tonoise $(S / N)$ ratios were sought.

\section{Combinatorial Optimization}

The aim of fitting experimental data to a regression model was to predict the chromatographic signal response for other IT-MS parameter combinations that were not tested in the laboratory. When the second order linear model (Equation 2) does not significantly fit data better than the first order one (Equation 1), the optimal matching corresponds to any of the extreme points of all allowed significant parameter values. Conversely, if the second order model fits better but the stationary points are not maxima or even if they are but do not match any possible combination of MS/MS parameters, it becomes necessary to screen all allowed combinations and to calculate the response in order to find the maximum $\mathrm{S} / \mathrm{N}$ ratio. For this purpose, a combinatorial optimization was carried out. A visual basic for applications macro in Microsoft Excel environment was created to evaluate the regression model equation for a maximum of $8 \mathrm{ET} \times 6 \mathrm{IT} \times 5 \mathrm{EV} \times 3 \mathrm{q} \times 3 \mathrm{IMW}$ combinations in a total of 2160 arrangements and save the best value. The maximum signal and optimal match of the MS/MS parameters are presented in Table 3.

Concerning the excitation voltage (EV) concerns, the value that maximizes the response when this parameter is significant was $0.2 \mathrm{~V}$, except for four organochlorine pesticides (aldrin, dieldrin, methoxychlor, and o,p'-DDT took the value of $2.0 \mathrm{~V}$ ) and several others where the EV had no significant effect on the signal response (e.g., lindane, azoxystrobin, $\beta$-cyfluthrin, tetraconazole, and iprodione). In general, the optimal ET value was $5 \mathrm{~ms}$ corresponding to the lowest allowed value except for some of the organochlorine pesticides for which a value of $60 \mathrm{~ms}$ was obtained. The "q" values that maximize the response were 0.30 and 0.45 . It can also be extracted from Table 3 that there is a strong correlation between ET and EV parameters. When both parameters are significant there is a linear positive relationship between them.

\section{Validation Process}

Analyte fortification was carried out by applying a known concentration of the 46 pesticides to a known quantity of blank strawberry. Following fortification, spiked samples were allowed to equilibrate $30 \mathrm{~min}$ at room temperature prior to extraction. Linearity of the analytical method described was studied using calibration curves with six

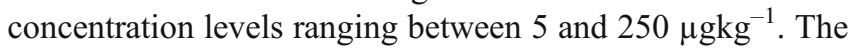
calibration curves were linear given that the determination coefficients were always higher than 0.99 for all pesticides. For all 46 studied pesticides, the LODs were lower than $12 \mu \mathrm{g} \mathrm{kg}^{-1}$. The results indicate that the LOD is adequate for food safety.

Recovery was studied in a spiked strawberry sample at three levels of concentration. The majority of the pesticides gave satisfactory recoveries (ranging from $70 \%$ to $120 \%$ ). Despite diazinon (60\%) tolylfluanid (62\%), and p,p'-DDE (59\%) in the lower spiking level, and mepanipyrim (139\%), myclobutanil (154\%), tetraconazole (131\%) and fludioxonil (132\%) in the higher spiking level, all others had satisfactory recoveries (in a range from $70 \%$ to $120 \%$ ) after QuEChERS and cleanup steps. 
Table 3. The Best MS/MS Significant Parameter Combinations

\begin{tabular}{|c|c|c|c|c|c|c|}
\hline & \multirow[b]{2}{*}{ Pesticides } & \multicolumn{5}{|c|}{ Best parameters by combinatorial optimization } \\
\hline & & ET & IT & EV & $\mathrm{q}$ & IMW \\
\hline Chloroacetanilide & Alachlor & 5 & 24 & 0.2 & - & 4 \\
\hline \multirow{11}{*}{ Organochlorines } & Aldrin & 60 & - & 2.0 & - & - \\
\hline & Dieldrin & 60 & - & 2.0 & 0.30 & 4 \\
\hline & Endrin & 60 & - & 2 & 0.30 & 1 \\
\hline & Hexachlorobenzene & - & 2 & 0.2 & 0.45 & - \\
\hline & Lindane & 60 & - & - & - & - \\
\hline & Methoxychlor & 60 & - & 2.0 & 0.30 & 1 \\
\hline & o,p'-DDT & 60 & 36 & 2.0 & 0.30 & - \\
\hline & $\mathrm{p}, \mathrm{p}^{\prime}-\mathrm{DDE}$ & 5 & 2 & 0.2 & 0.45 & 2 \\
\hline & $\alpha$-Endosulfan & 5 & 2 & 0.2 & 0.30 & - \\
\hline & $\beta$-Endosulfan & - & 2 & 0.2 & 0.30 & - \\
\hline & IS & 5 & 2 & 0.2 & 0.45 & - \\
\hline \multirow[t]{3}{*}{ Triazines } & Atrazin & 5 & 12 & 0.2 & - & - \\
\hline & Atrazine-desethyl & 5 & 12 & 0.2 & - & - \\
\hline & Simazine & - & - & 0.2 & - & - \\
\hline Strobin & Azoxystrobin & 5 & 2 & - & - & - \\
\hline \multirow{8}{*}{ Pyrethroids } & $\beta$-Cyfluthrin & - & 2 & - & - & - \\
\hline & Bifentrin & 5 & - & 0.2 & 0.30 & - \\
\hline & Cypermethrin & - & - & - & 0.45 & - \\
\hline & Deltametrin & 5 & - & 0.2 & - & 1.0 \\
\hline & Fenpropathrin & 5 & - & 0.2 & 0.45 & 4 \\
\hline & Fenvalerate & 5 & 12 & 0.2 & - & - \\
\hline & $\lambda$-Cyhalotrhin & 5 & - & 0.2 & 0.45 & 4 \\
\hline & Permethrin & - & 2 & 0.2 & 0.30 & 4 \\
\hline \multirow[t]{4}{*}{ Pyrimidine } & Bupirimate & 5 & 24 & 0.2 & - & - \\
\hline & Mepanipyrim & - & - & - & - & - \\
\hline & Cyprodinil & 5 & 12 & - & - & - \\
\hline & Pyrimethanil & - & 12 & - & - & - \\
\hline \multirow[t]{2}{*}{ Thiophthalimide } & Captan & - & 2 & - & - & - \\
\hline & Folpet & 5 & 24 & 0.2 & - & 1.0 \\
\hline \multirow{3}{*}{ Organophosphorus } & Chlorpyrifos & - & - & 0.2 & 0.30 & - \\
\hline & Diazinon & 5 & - & 0.2 & - & - \\
\hline & Malathion & 5 & 12 & 0.2 & - & - \\
\hline \multirow[t]{2}{*}{ Unclassified } & Dazomet & - & 24 & - & - & 2 \\
\hline & Fludioxinil & 5 & 24 & 0.2 & 0.45 & - \\
\hline Anilide & Fenhexamid & - & 2 & - & - & - \\
\hline \multirow[t]{2}{*}{ Aryloxyphenoxy propionic acid } & Fluazifop-p-butyl & 5 & 12 & 0.2 & - & 4 \\
\hline & Quizalofop-p-ethyl & 5 & - & - & - & - \\
\hline$N$-methyl carbamate & Methiocarb & - & - & 0.2 & - & - \\
\hline \multirow[t]{2}{*}{ Azole } & Myclobutanil & - & 24 & 0.2 & - & - \\
\hline & Tetraconazole & 5 & - & - & - & - \\
\hline 2,6-Dinitroaniline & Pendimethalin & 5 & 2 & 0.2 & - & - \\
\hline Phenylsulfamide & Tolylfluanid & 5 & - & 0.2 & - & - \\
\hline \multirow[t]{3}{*}{ Dicarboximide } & Vinclozolin & - & - & - & - & - \\
\hline & Procymidone & 5 & 12 & 0.2 & - & - \\
\hline & Iprodione & 5 & 24 & - & - & - \\
\hline
\end{tabular}

Default parameter

\section{Screening of Unknown Samples}

The optimized method using the 46 multiclass pesticide screening program was applied to Portuguese strawberry samples. In each unknown sample, the target (quantifier) and qualifier MRM transitions of each test compound (Table 1) were monitored. Five percent of all samples examined contained levels of organochlorine pesticides greater than the limit established by the European Union for strawberry samples. The highest value was $27.30 \mu \mathrm{g} \cdot \mathrm{kg}^{-1}$ for lindane [5].

In only three samples, residues of fludioxonil, bifenthrin, mepanipyrim, tolylfluanid, cyprodinil, tetraconazole, iprodione, and malathion were detected, and the concentrations were much lower than their harmonized EU MRL. The highest concentration was obtained for iprodione (1071 $\mu \mathrm{g} . \mathrm{kg}^{-1}$ ), which is lower, however, than the MRL value. Looking for positive pesticide residues, except for organochlorine pesticides that are stable and very persistent in the environment, most of the others are commonly used in strawberries crops.

\section{Conclusions}

The selected optimization strategy based on multiple linear regression allows for an efficient method development. It is not only helpful to optimize all compounds or a group of them, as it can also help to establish the optimum values for 
all parameters of the MS/MS system. In the case of OCPs (aldrin, dieldrin, endrin, lindane, and methoxychlor) that tend to be very persistent in the environment, it is proposed that because of their stable structure, higher ET $(60 \mathrm{~ms})$ and $\mathrm{EV}(2 \mathrm{v})$ are required to achieve the best signal response. The results showed that certain pesticides, which have low signal in MS/MS, also showed poorly to fit in the models. The applicability of the method was demonstrated by analysis of real samples (strawberries), resulting in good quality control data, thus making possible rapid and reliable determination of the targeted pesticides.

This paper shows that the statistics study is a useful tool to optimize this kind of analytical parameters. Particularly, multiple linear regression was an important tool to predict the "best" combination of IT-MS parameters to maximize the analytical response within the range of values experimentally tested in this work.

\section{Acknowledgment}

This research was supported by a Ph.D. grant (BD/47200/ 2008) from Fundação para a Ciência e a Tecnologia, cofinanced by POPH/FSE (Programa Operacional Potencial Humano/Fundo Social Europeu), grant no. PEst-C/EQB/ LA0006/201 and Ph.D. grant from programa Mundus.

\section{References}

1. Cervera, M.I., Medina, C., Portoles, T., Pitarch, E., Beltran, J., Serrahima, E., Pineda, L., Munoz, G., Centrich, F., Hernandez, F.: Multi-residue determination of 130 multiclass pesticides in fruits and vegetables by gas chromatography coupled to triple quadrupole tandem mass spectrometry. Anal. Bioanal. Chem. 397, 2873-2891 (2010)

2. Banerjee, K., Savant, R.H., Dasgupta, S., Patil, S.H., Oulkar, D.P., Adsule, P.G.: Multiresidue analysis of synthetic pyrethroid pesticides in grapes by gas chromatography with programmed temperature vaporizing-large volume injection coupled with ion trap mass spectrometry. J. AOAC Int. 93, 368-379 (2010)

3. Regulation (EC) No $396 / 2005$ of the European parliament and of the council of 23 February 2005 on maximum residue levels of pesticides in or on food and feed of plant and animal origin and amending Council Directive 91/414/EEC. (2005)

4. Amvrazi, E.G., Tsiropoulos, N.G.: Application of single-drop microextraction coupled with gas chromatography for the determination of multiclass pesticides in vegetables with nitrogen phosphorus and electron capture detection. J. Chromatogr. A. 1216, 2789-2797 (2009)

5. Fernandes, V.C., Domingues, V.F., Mateus, N., Delerue-Matos, C.: Organochlorine pesticide residues in strawberries from integrated pest management and organic farming. J. Agric. Food Chem. 59, 7582-7591 (2011)

6. Liu, X., Wang, X., Xu, J., Dong, F., Song, W., Zheng, Y.: Determination of tebuconazole, trifloxystrobin and its metabolite in fruit and vegetables by a Quick, Easy, Cheap, Effective, Rugged and
Safe (QuEChERS) method using gas chromatography with a nitrogenphosphorus detector and ion trap mass spectrometry. Biomed. Chromatogr. 25, 1081-1090 (2011)

7. Fernandes, V.C., Domingues, V.F., Mateus, N., Delerue-Matos, C.: Determination of pesticides in fruit and fruit juices by chromatographic methods. An overview. J. Chromatogr. Sci. 49, 715-730 (2011)

8. Beltran, J., Peruga, A., Pitarch, E., Lopez, F.J., Hernandez, F.: Application of solid-phase microextraction for the determination of pyrethroid residues in vegetable samples by GC-MS. Anal. Bioanal. Chem. 376, 502-511 (2003)

9. Hunter, R.E., Riederer, A.M., Ryan, P.B.: Method for the determination of organophosphorus and pyrethroid pesticides in food via gas chromatography with electron-capture detection. J. Agric. Food Chem. 58, 1396-1402 (2010)

10. Lambropoulou, D.A., Psillakis, E., Albanis, T.A., Kalogerakis, N.: Single-drop microextraction for the analysis of organophosphorous insecticides in water. Anal. Chim. Acta 516, 205-211 (2004)

11. Huskova, R., Matisova, E., Kirchner, M.: Fast GC-MS pesticide multiresidue analysis of apples. Chromatographia 68, S49-S55 (2008)

12. Vidal, J.L.M., Arrebola, F.J., Mateu-Sanchez, M.: Multi-residue method for determination of pesticides in vegetable samples by GC-MS-MS. Chromatographia 56, 475-481 (2002)

13. Castillo, M., Gonzalez, C., Miralles, A.: An evaluation method for determination of non-polar pesticide residues in animal fat samples by using dispersive solid-phase extraction clean-up and GC-MS. Anal. Bioanal. Chem. 400, 1315-1328 (2011)

14. Mandalakis, M., Tsapakis, M., Stephanou, E.G.: Optimization and application of high-resolution gas chromatography with ion trap tandem mass spectrometry to the determination of polychlorinated biphenyls in atmospheric aerosols. J. Chromatogr. A. 925, 183-196 (2001)

15. Lehotay, S.J., Gates, R.A.: Blind analysis of fortified pesticide residues in carrot extracts using GC-MS to evaluate qualitative and quantitative performance. J. Sep. Sci. 32, 3706-3719 (2009)

16. Koesukwiwat, U., Lehotay, S.J., Leepipatpiboon, N.: Fast, low-pressure gas chromatography triple quadrupole tandem mass spectrometry for analysis of 150 pesticide residues in fruits and vegetables. $J$. Chromatogr. A. 1218, 7039-7050 (2011)

17. Naert, C., De Saeger, S., Van Peteghem, C.: Development of a gas chromatography/ion trap mass spectrometry based method for the quantification of polybrominated diphenyl ethers and polychlorinated biphenyls in adipose tissue. Rapid Comm. Mass Spectrom. 18, 2317$2322(2004)$

18. Yusa, V., Pardo, O., Pastor, A., de la Guardia, M.: Optimization of a microwave-assisted extraction large-volume injection and gas chromatography-ion trap mass spectrometry procedure for the determination of polybrominated diphenyl ethers, polybrominated biphenyls and polychlorinated naphthalenes in sediments. Anal. Chim. Acta 557, 304-313 (2006)

19. Leon, N., Yusa, V., Pardo, O., Pastor, A.: Determination of 3-MCPD by GC-MS/MS with PTV-LV injector used for a survey of Spanish foodstuffs. Talanta 75, 824-831 (2008)

20. Uveges, M., Abranko, L., Fodor, P.: Optimization of GC-ICPMS system parameters for the determination of butyltin compounds in Hungarian freshwater origin sediment and mussel samples. Talanta 73, 490-497 (2007)

21. Rao, C.R., Linear statistical inference and its applications. In 2nd ed.; John Wiley \& Sons, Inc.: New York (1973)

22. Horn, R.: Statistical-Methods for model discrimination - Applications to gating kinetics and permeation of the acetylcholine-receptor channel. Biophys. J. 51, 255-263 (1987) 